\title{
Helium ions put magnetic skyrmions on the
}

\section{track}

Roméo Juge, ${ }^{*, \dagger}, \#$ Kaushik Bairagi, ${ }^{\dagger}$ Kumari Gaurav Rana, ${ }^{\dagger}$ Jan Vogel, ${ }^{\ddagger}$ Mamour Sall, ${ }^{\top}$ Dominique Mailly, ${ }^{\S}$ Van Tuong Pham, ${ }^{\dagger}$ Qiang Zhang, ${ }^{\dagger}$ Naveen Sisodia, ${ }^{\dagger}$ Michael Foerster," Lucia Aballe,, Mohamed Belmeguenai, ${ }^{\perp}$ Yves Roussigné, ${ }^{\perp}$ Stéphane Auffret, ${ }^{\dagger}$ Liliana Buda-Prejbeanu $^{\dagger}$ Gilles Gaudin $^{\dagger}{ }^{\text {Dafiné Ravelosona, }}$ and Olivier Boulle*,†

$\dagger$ †niv. Grenoble Alpes, CNRS, CEA, Grenoble INP, Spintec, 38000 Grenoble, France $\ddagger$ Univ. Grenoble Alpes, CNRS, Institut Néel, 38000 Grenoble, France

【Spin-Ion Technologies, 91120 Palaiseau, France

$\S$ Centre de Nanosciences et de Nanotechnologies, CNRS, Université Paris-Saclay, 91120

Palaiseau, France

॥ALBA Synchrotron Light Facility, 08290 Cerdanyola del Vallès, Barcelona, Spain

$\perp$ Laboratoire des Sciences des Procedés et des Matériaux, CNRS, Université Sorbonne

Paris Nord, 93430 Villetaneuse, France

\#Present address: Spin-Ion Technologies, 91120 Palaiseau, France

E-mail: romeo.juge@spin-ion.com; olivier.boulle@cea.fr

\begin{abstract}
Magnetic skyrmions are deemed to be the forerunners of novel spintronic memory and logic devices. While their observation and their current-driven motion at room temperature have been demonstrated, certain issues regarding their nucleation, stability,
\end{abstract}


pinning and skyrmion Hall effect still need to be overcome to realise functional devices. Here we demonstrate that focused $\mathrm{He}^{+}$-ion-irradiation can be used to create and guide skyrmions in racetracks. We show that the weakening of the perpendicular magnetic anisotropy and Dzyaloshinskii-Moriya interaction in the track defined by ion-irradiation leads to the formation of stable isolated skyrmions. Current-driven skyrmion motion experiments and simulations reveal that the skyrmions move along the irradiated track, resulting in a suppression of the skyrmion Hall effect, and that the skyrmion velocity and confinement can be enhanced by tuning the magnetic properties. These results open up a new path to nucleate and guide skyrmions in racetrack devices.

\section{Keywords}

magnetic skyrmions, ion-irradiation, magnetic patterning, racetrack, current-induced dynamics, skyrmion Hall effect

Magnetic skyrmions are topological magnetic textures that hold promise for applications in spintronic devices owing to their nanometric size and particle-like properties. ${ }^{1,2}$ This has steered intensive research leading to their observation at room temperature and their

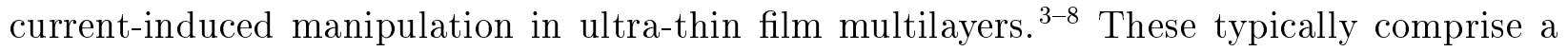
heavy metal, a nm-thick magnetic material and a non-magnetic layer, combining the essential perpendicular magnetic anisotropy (PMA) and Dzyaloshinskii-Moriya interaction (DMI) for the skyrmion stability, as well as spin-orbit torques (SOTs) for their current-driven motion. $3,6,9,10$

The most prominent skyrmion-based applications such as racetrack memory ${ }^{11}$ and logic devices ${ }^{12-16}$ rely on the stabilisation and controlled shift of trains of skyrmions by current pulses inside narrow magnetic tracks. While the demonstration of their current-driven motion in $\mu$ m-wide tracks at room temperature was a first important step, ${ }^{6,9,17}$ the effective realisation of such racetrack devices still faces important bottlenecks. Firstly, moving skyrmions are subject to a gyrotropic force due to their non-trivial topology, resulting in a 
transverse motion toward the edge of the track. This so-called skyrmion Hall effect (SkHE) is a major issue as it can lead to the skyrmion annihilation on the track edge. ${ }^{17-19}$ Secondly, reliable skyrmion motion in tracks is impeded by pinning and uncontrolled skyrmion nucleation/annihilation events, which can result from local film inhomogeneities ${ }^{17,20,21}$ as well the roughness on the track edges created by the lithography and etching processes. ${ }^{18,22-24}$ The latter is a critical issue for tracks with nanoscale lateral dimensions targeted for highdata-density applications. Finally, the large stray field emanating from the edges can also significantly affect the skyrmion size and stability and prevent dense packing of parallel racetracks.

To solve these issues, a promising approach is to pattern skyrmion racetracks by local modification of the magnetic properties. The idea is to define a magnetic potential well that will help stabilising the skyrmions as well as guide their motion, leading to faster and SkHE-free dynamics ${ }^{25-40}$ combined with reduced pinning. This can be achieved by local ion-irradiation, ${ }^{41-43}$ and in particular light-ion-irradiation such as $\mathrm{He}^{+}$. Unlike heavier ions, $\mathrm{He}^{+}$ions (in the $10 \mathrm{keV}$ energy range) traverse the stack inducing short-range atomic displacements and implant deep into the substrate. ${ }^{44}$ This leads to a gentle modification of the magnetic properties, preventing harsh material damage such as surface sputtering ${ }^{45}$ and cascade collisions. This technique was shown to modify the magnetic properties through soft intermixing in typical ultra-thin trilayers. ${ }^{41,46-48}$ More specifically, the PMA and DMI, of interfacial origin in these ultra-thin films, can be finely tuned by playing on the irradiation dose. ${ }^{45-47,49,50}$ Spatial modulation of the magnetic properties can be achieved by irradiating through a mask ${ }^{41}$ or by using a focused ion beam (FIB). ${ }^{42}$ It can thus be used as a method to pattern the magnetic properties without physical etching that introduce defects at the edges of the nanostructures. It was recently used to nucleate isolated skyrmions in different confined geometries such as dots defined by local ion-irradiation, ${ }^{38,51-53}$ although the confinement precluded any manipulation of the nucleated skyrmions. In addition, $\mathrm{He}^{+}$-irradiation can actually reduce the pre-existing pinning centre density through interface smoothing. ${ }^{48,54,55}$ 
In this work, we investigated the skyrmion nucleation and their channelled current-driven motion at room temperature in $\mathrm{Pt} / \mathrm{Co} / \mathrm{MgO}$ racetracks patterned by $\mathrm{He}^{+}$-irradiation. This stack is known to exhibit large interfacial DMI and to host stable, homochiral Néel skyrmions at room temperature. ${ }^{5}$ Magnetic microscopy experiments reveal that the weakening of the anisotropy and DMI in the irradiated tracks leads to the formation of stable, sub-50 nm isolated magnetic skyrmions. Moreover, current-driven skyrmion motion experiments and simulations show that the skyrmions move along the track defined by $\mathrm{He}^{+}$-irradiation, resulting in a suppression of the SkHE. Micromagnetic simulations and analytical models allow us to identify the material properties in order to enhance the skyrmion velocity and confinement in the tracks. These results open up a new route to nucleate and guide magnetic skyrmions in racetrack memory and logic devices.

We first investigated full film samples consisting of $\mathrm{Ta}(3) / \mathrm{Pt}(3) / \mathrm{Co}(0.97) / \mathrm{MgO}(0.9) /$ $\mathrm{Ta}(2)$ (thicknesses in $\mathrm{nm}$ ) to get an insight into how $\mathrm{He}^{+}$-irradiation affects the magnetic properties of this stack. The films were deposited at room temperature by DC magnetron sputtering on $\mathrm{Si} / \mathrm{SiO}_{2}$ substrates. They were then uniformly irradiated at room temperature (using a Helium-S ${ }^{\circledR}$ system from Spin-Ion Technologies) with a $15 \mathrm{keV} \mathrm{He}^{+}$ion beam at different ion fluences. Fig. 1(a) and (b) show respectively the anisotropy field and the DMI constant measured in the irradiated films. These were measured from hard-axis loops by vibrating sample magnetometry (VSM) and by Brillouin light scattering (BLS) spectroscopy ${ }^{56}$ respectively (see Supporting Information). As the fluence increases, we observe that the PMA decreases monotonically, while a decrease of the DMI is observed at low fluence followed by a slight increase at larger fluence.

When traversing the material stack, the ions induce short-range atomic displacements in the order of a few inter-atomic distances, leading to interface intermixing and hence altering the interface-induced PMA and DMI. ${ }^{41,43}$ In particular, it was found that the Pt/Co interface roughness, in other words intermixing, increases linearly with irradiation fluence, resulting in a continuous decrease of interfacial PMA. ${ }^{57}$ Regarding the DMI, a similar decrease of the 

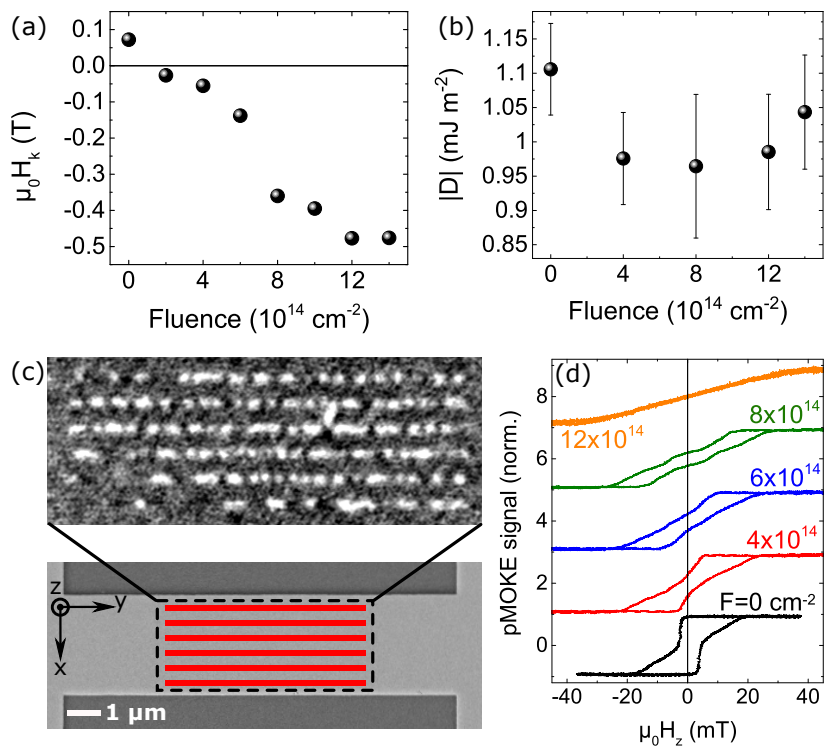

Figure 1: (a) Anisotropy field, defined as the hard-axis saturation field measured by VSM, and (b) DMI constant, extracted from BLS experiments, measured in $\mathrm{Pt} / \mathrm{Co} / \mathrm{MgO}$ extended films irradiated at different fluences with a mm-size $\mathrm{He}^{+}$-ion beam (see Supporting Information). (c) XMCD-PEEM (top) and SEM (bottom) images of a 3- $\mu$ m-wide Pt/Co/MgO wire. The red tracks, of dimensions $6000 \times 150 \mathrm{~nm}^{2}$ with a spacing of $300 \mathrm{~nm}$, indicate regions irradiated using a $\mathrm{He}^{+}$FIB. The XMCD-PEEM image of the inset shows the magnetic configuration at $\mu_{0} H_{z} \approx 10 \mathrm{mT}$ and for a fluence $6 \times 10^{14} \mathrm{~cm}^{-2}$. (d) pMOKE hysteresis loops measured in $2 \times 2 \mathrm{\mu m}^{2}$ irradiated areas of the sample treated by FIB (c) using a sub- $\mathrm{mm}$ focused laser spot. Note that, although nominally identical, the patterned sample (c,d) had a slightly larger PMA pre-irradiation than the full film sample where $\mu_{0} H_{K}$ and $D$ were measured $(a, b)$. Therefore, a larger fluence is needed to bring the magnetisation in the plane: $12 \times 10^{14} \mathrm{~cm}^{-2}$ in (d) against $2 \times 10^{14} \mathrm{~cm}^{-2}$ in (a).

DMI with the $\mathrm{He}^{+}$-irradiation dose was reported in $\mathrm{W} / \mathrm{FeCoB} / \mathrm{MgO}$ ultra-thin films, ${ }^{48}$ although an increase was reported in $\mathrm{Ta} / \mathrm{CoFeB} / \mathrm{MgO}^{47,58}$ and $\mathrm{Ta} / \mathrm{CoFeB} / \mathrm{Pt}^{50}$ films. Ab initio calculations predict that intermixing at the $\mathrm{Pt} / \mathrm{Co}$ interface results in a slight diminution of DMI, ${ }^{59}$ around 20\%, in good agreement with our experiments (Fig. 1(b)).

We note that the $\mathrm{Co} / \mathrm{MgO}$ interface is expected to be more robust against intermixing than the Pt/Co interface. Indeed, $\mathrm{Pt}$ and $\mathrm{Co}$ have a negative enthalpy of mixing while that of $\mathrm{Mg}$ and Co is positive. ${ }^{60}$ Co will therefore preferentially mix with Pt than with $\mathrm{Mg}$. Hence, the effect of ion-irradiation on the magnetic properties at the $\mathrm{Co} / \mathrm{MgO}$ interface may be neglected as a first approximation.

We then studied the effect of local $\mathrm{He}^{+}$-irradiation in a similar $\mathrm{Pt} / \mathrm{Co} / \mathrm{MgO}$ film. The 


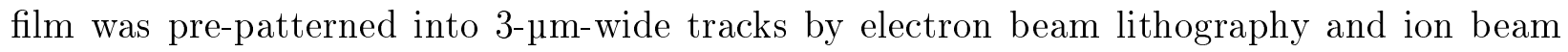
etching. It was then irradiated with a $\mathrm{He}^{+} \mathrm{FIB}$ at constant ion energy $(25 \mathrm{keV}$ ), which allows us to control the geometry of the irradiated areas with a high spatial resolution (down to a few nm). Fig. 1(c) displays a scanning electron microscopy (SEM) image of a $\mathrm{Pt} / \mathrm{Co} / \mathrm{MgO}$ track with 150-nm-wide irradiated tracks marked in red. The image on top, acquired by X-ray magnetic circular dichroism photo-emission electron microscopy (XMCDPEEM), ${ }^{61,62}$ displays the magnetic configuration in the presence of an external out-of-plane (OOP) magnetic field $\mu_{0} H_{z} \approx 10 \mathrm{mT}$. We observe skyrmions and elongated stripes aligned along the tracks while the pristine area is uniformly magnetised. Fig. 1(d) displays the OOP hysteresis loops measured for different fluences ranging from 0 (pristine) to $12 \times 10^{14} \mathrm{~cm}^{-2}$. The loops highlight the gradual softening of the magnetic layer with increasing fluence up

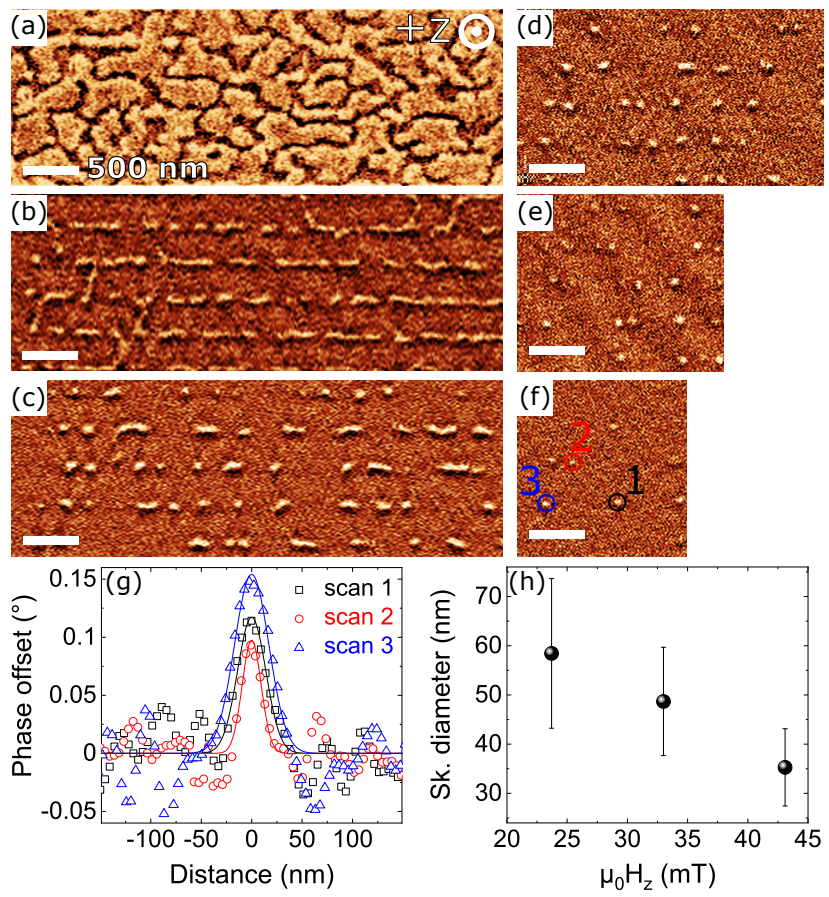

Figure 2: (a-f) MFM images showing the magnetic texture in the irradiated tracks of Fig. 1(c) at different external out-of-plane magnetic fields: (a) $\mu_{0} H_{z}=0 \mathrm{mT}$, (b) $10 \mathrm{mT}$, (c) 19 $\mathrm{mT}$, (d) $24 \mathrm{mT}$, (e) $33 \mathrm{mT}$, (f) $43 \mathrm{mT}$. In (a), the magnetisation was saturated beforehand by a large field $\mu_{0} H_{z} \approx-100 \mathrm{mT}$. (g) Line-scans of the MFM signal (symbols) acquired for the three skyrmions in (f). Solid lines are Gaussian fits. (h) Skyrmion diameter, defined as the full width at half maximum of the Gaussian, as a function of the external field and averaged over multiple skyrmions in images (d-f). 
Table 1: Experimental parameters used in the micromagnetic simulations. The saturation magnetisation $\left(M_{s}\right)$ and the anisotropy field $\left(\mu_{0} H_{K}\right)$ were extracted from VSM measurements. A higher $\mu_{0} H_{K}$ in the non-irradiated zone was also used to study its effect on the dynamics. The DMI constant $(D)$ was extrapolated from the BLS measurements presented in Fig. 1(b). The exchange constant $(A)$ and the magnetic damping parameter $(\alpha)$ were taken from a previous study in the same system. ${ }^{17}$ A lower $\alpha$ was also used to study its effect on the dynamics.

\begin{tabular}{cccccc}
\hline & $\begin{array}{c}M_{s} \\
\left(\mathrm{MA} \mathrm{m}^{-1}\right)\end{array}$ & $\begin{array}{c}\mu_{0} H_{K} \\
(\mathrm{mT})\end{array}$ & $\begin{array}{c}|D| \\
\left(\mathrm{mJ} \mathrm{m}^{-2}\right)\end{array}$ & $\begin{array}{c}A \\
\left(\mathrm{pJ} \mathrm{m}^{-1}\right)\end{array}$ & $\alpha$ \\
\hline Non-irradiated & 1.32 & 72 & 1.122 & 16 & 0.3 \\
Irradiated & 1.32 & 7 & 1.082 & 16 & 0.3 \\
\hline
\end{tabular}

to a point where the magnetisation turns in-plane $\left(12 \times 10^{14} \mathrm{~cm}^{-2}\right)$.

We now look closely at the magnetisation texture in the irradiated tracks of Fig. 1(c). Fig. 2(a-f) show magnetic force microscopy (MFM) images acquired for different OOP external fields. At $H_{z}=0$ (Fig. 2(a)), the image displays labyrinthine worm domains. Then, upon increasing the external field, the bright domains progressively shrink, up to a point where the pristine zone becomes uniformly magnetised (Fig. 2(b)), revealing stripe domains well aligned in the irradiated tracks. Increasing further the field (Fig. 2(c-f)) leads to the separation of these worms into a single array of isolated skyrmions, that are then compressed towards smaller sizes. In the pristine zone, on the contrary, the large magnetic domains do not shrink into isolated skyrmions when increasing the external field due to the larger anisotropy, as indicated by the non-zero remanence in the zero-fluence loop of Fig. 1(d). Furthermore, skyrmions can be compressed to very small sizes. Fig. 2(g) shows line-scans of the MFM signal (symbols) acquired at $\mu_{0} H_{z}=43 \mathrm{mT}$ for the three skyrmions marked in Fig. 2(f) with Gaussian fit (solid lines), which gives the full width at half maximum $d_{s k 1}=33 \mathrm{~nm}, d_{s k 2}=22 \mathrm{~nm}$ and $d_{s k 3}=41 \mathrm{~nm}$. Using this definition, we plot in Fig. $2(\mathrm{~h})$ the skyrmion diameter as a function of the external field extracted from Fig. 2(d-f), which reaches an average of $35 \mathrm{~nm}$ at $\mu_{0} H_{z}=43 \mathrm{mT}$.

To get more insight into these experimental results, we performed micromagnetic simu- 
lations with Mumax $3^{63}$ using the experimental parameters listed in Table 1 . We considered a simulation window of dimensions $500 \times 500 \times 0.9 \mathrm{~nm}^{3}$ where $\mathrm{x}$ and $\mathrm{y}$ periodic boundary conditions were imposed to mimic the experimental conditions. Then, we simulated the ion-irradiated tracks by a 150-nm-wide region with lower PMA and DMI. In the simulations, we start from a labyrinthine worm domain configuration and let it relax at zero external field (Fig. 3(a)). We observe that the worm domains spread over both the non-irradiated and the irradiated regions, in accordance with the MFM observations. Then, as the external OOP field is increased, the worm domains shrink along the irradiated region with reduced PMA and DMI, acquiring an elongated shape along $y$, while the non-irradiated region saturates due to its larger anisotropy (Fig. 3(b)). Upon further increase of the OOP field, the skyrmions shrink and acquire a circular shape. This is explained by a reduced influence of the confinement potential when the skyrmions become smaller than the track width (Fig. 3(c) and (d)). Fig. 3(e) shows the evolution of the skyrmion size along $x$ and $y$ as a function of the external field, in line with our experimental findings (Fig. 2(h)). We also studied the effect of the irradiation fluence, i.e. anisotropy and DMI values, on the skyrmion size (Fig. $3(\mathrm{f}))$ : the skyrmion diameter increases with irradiation fluence since the domain wall energy is reduced at larger fluence due to the lower anisotropy and DMI (see Fig. 1(a) and (b)).

The simulations also allow us to investigate the current-driven dynamics of the magnetic skyrmions in the irradiation-defined racetracks. We only considered the damping-like SOT (DL-SOT) since the effect of the field-like SOT is negligible in our system. ${ }^{17}$ The DL-SOT reads $\boldsymbol{T}_{D L}=-\mu_{0} \gamma C_{D L} J \boldsymbol{m} \times[(\hat{\boldsymbol{z}} \times \hat{\boldsymbol{j}}) \times \boldsymbol{m}]$, with $\gamma$ the gyromagnetic ratio, $\boldsymbol{m}$ the reduced magnetisation vector, $\hat{j}$ the unit vector in the current direction and $C_{D L}$ the effective field per unit current density associated with the DL-SOT. We take $C_{D L}=2.1 \times 10^{-14} \mathrm{~T} \mathrm{~A}^{-1}$ $\mathrm{m}^{2}$ from previous experiments. ${ }^{17}$ Fig. 4(a) shows successive snapshots of a skyrmion moving after the injection of a current density $J=6.6 \times 10^{10} \mathrm{~A} \mathrm{~m}^{-2}$ along $+y$. In the first few ns, the injected current leads to a skyrmion motion along the track direction $(y)$ due to the DL-SOT-induced driving force, as well as a transverse motion toward the edge induced 

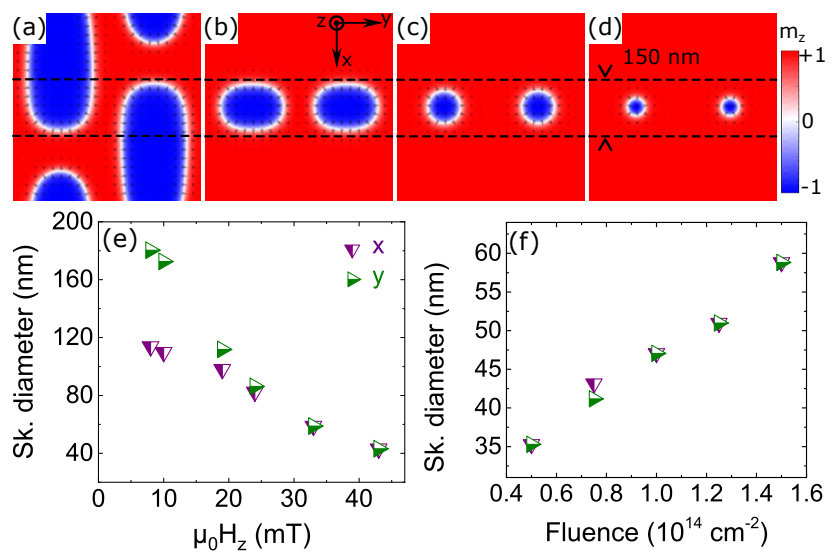

Figure 3: Micromagnetic simulations. Skyrmion stabilisation in a 150-nm-wide irradiated track (region between the dotted lines) at a fluence $1.5 \times 10^{14} \mathrm{~cm}^{-2}$ and at different external magnetic fields, applied opposite to the skyrmion core magnetisation: (a) $\mu_{0} H_{z}=0 \mathrm{mT}$, (b) $8 \mathrm{mT}$, (c) $19 \mathrm{mT}$ and (d) $33 \mathrm{mT}$. The window size is $500 \times 500 \mathrm{~nm}^{2}$ with a grid of $512 \times 512 \times 1$ cells and periodic boundary conditions on both the $x$ and the $y$ direction. (e) Skyrmion size along $x$ and $y$ as a function of the external field for a fluence $1.5 \times 10^{14} \mathrm{~cm}^{-2}$ and (f) as a function of the fluence for $\mu_{0} H_{z}=33 \mathrm{mT}$.

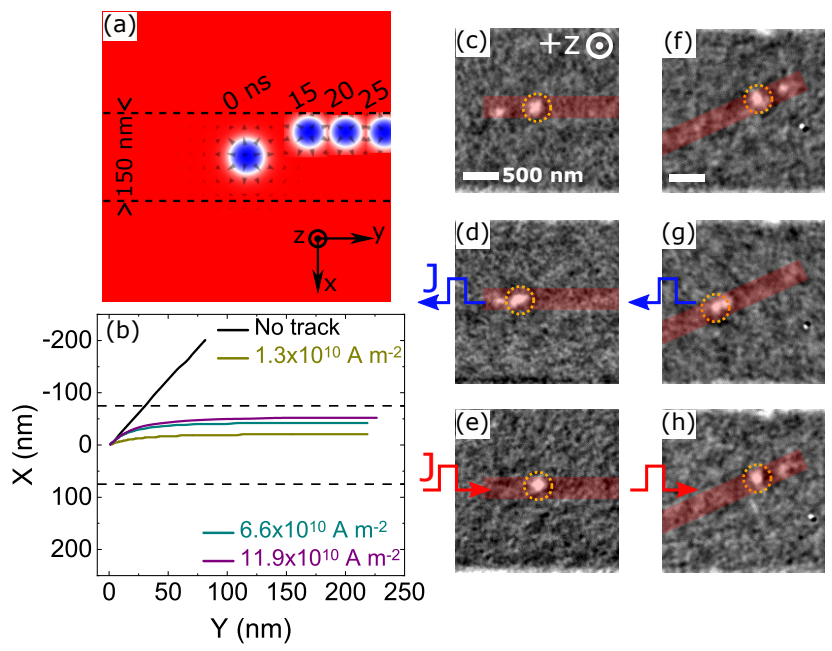

Figure 4: (a) Simulated current-driven skyrmion motion in a 150-nm-wide irradiated track (region between the dashed lines). The current flows along $+y$, the current density is $J=$ $6.6 \times 10^{10} \mathrm{~A} \mathrm{~m}^{-2}$ and the external OOP field is $\mu_{0} H_{z}=33 \mathrm{mT}$. We assume that the current is flowing in both the irradiated and non-irradiated areas, leading to a spatially homogeneous SOT in the magnetic track, and only considered the damping-like SOT (the filed-like SOT was neglected). ${ }^{17}$ (b) Skyrmion trajectories recorded at different current densities. (c-h) Sequence of XMCD-PEEM images showing the current-driven motion of skyrmions in $\mathrm{He}^{+}$ ion-irradiated $\mathrm{Pt} / \mathrm{Co} / \mathrm{MgO}$ tracks (red shaded area) at an angle (c-e) $0^{\circ}$ and (f-h) $22.5^{\circ}$ with respect to the current direction. Between each image, a single $11 \mathrm{~ns}$ current pulse of amplitude $J=6.6 \times 10^{11} \mathrm{~A} \mathrm{~m}^{-2}$ is injected. The external field is $\mu_{0} H_{z} \approx 10 \mathrm{mT}$. 

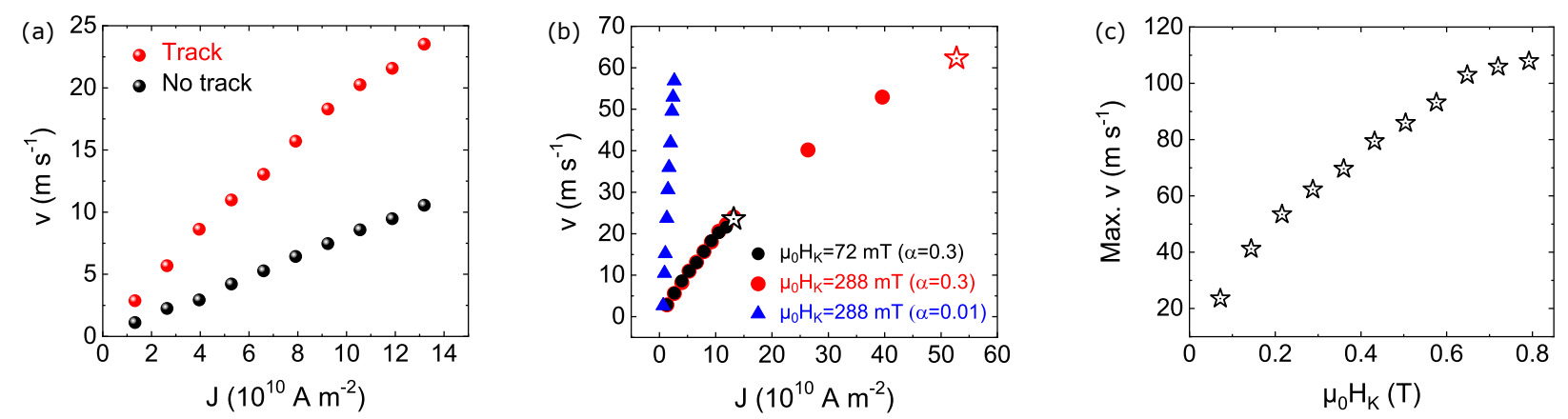

Figure 5: (a) Skyrmion velocity as a function of the current density with and without irradiated track where $\mu_{0} H_{K}=7 \mathrm{mT}$ inside $\left(\mu_{0} H_{K}=72 \mathrm{mT}\right.$ outside $)$ the track. In the absence of track, $\mu_{0} H_{K}=7 \mathrm{mT}$ everywhere. (b) Skyrmion velocity as a function of the current density for different anisotropies outside the irradiated track and different damping values $\alpha$. The black and red stars correspond to the maximum velocities that can be reached in each scenario. (c) Maximum skyrmion velocity as a function of the anisotropy field outside the track. The external field, opposite to the skyrmion core magnetisation, is $\mu_{0} H_{z}=33 \mathrm{mT}$ in all cases. For each current density, the steady-state velocity is calculated at a moment when the skyrmion is moving in a straight line along the edge of the ion-defined track (i.e. along $y$ ).

by the gyrotropic force (SkHE). The left-handed Néel chirality (imposed by the sign of the DMI), the skyrmion core polarity (set by the external field direction) and the sign of the spin Hall angle (given by the heavy metal layer), dictate a longitudinal motion along the current direction $(y)$ and a deflection along $-x \cdot{ }^{9,17}$ After 15 ns, the skyrmion reaches the track edge. The energy barrier at the track edge leads to a force opposite to the gyrotropic force, suppressing the transverse skyrmion motion, such that the skyrmion starts sliding along the edge, without SkHE. ${ }^{26,27,64}$ In the absence of track, i.e. assuming that the whole area is irradiated, the skyrmion moves in the direction imposed by the SkHE (see Fig. 4(b)): the larger the current density, the larger the deflection and the faster the skyrmion reaches the edge.

In Fig. 4(c-h), we show experimental results illustrating the guiding of the skyrmion motion: The images show the current-driven motion of a few skyrmions in irradiated tracks (area in red) at two different angles with respect to the current direction: $0^{\circ}$ (Fig. 4(c-e)) and $22.5^{\circ}$ (Fig. $4(\mathrm{f}-\mathrm{h})$ ). For each sequence of images, between each acquisition, a single 11 ns current pulse of amplitude $J=6.6 \times 10^{11} \mathrm{~A} \mathrm{~m}^{-2}$ is injected in the direction indicated by 
the arrow. Remarkably, the skyrmions move back and forth within the irradiated racetrack, at velocities of $v\left(0^{\circ}\right) \approx 35 \mathrm{~m} \mathrm{~s}^{-1}$ and $v\left(22.5^{\circ}\right) \approx 60 \mathrm{~m} \mathrm{~s}^{-1}$ along the track, while a significant SkHE is expected at these velocities. ${ }^{17}$

To get a deeper insight into the dynamics, we simulated the steady-state velocities of the moving skyrmions. Fig. 5(a) shows the skyrmion velocity as a function of the current density for the scenarios with and without track: the presence of the track not only guides the skyrmion motion, suppressing their transverse velocity, but also enhances their longitudinal velocity. The energy barrier at the track edge creates a repulsive force, thereby cancelling the gyrotropic force, which in turn helps in enhancing the longitudinal velocity. ${ }^{9,26,33,64}$ Note however that above some threshold current density/velocity $\left(1.3 \times 10^{11} \mathrm{~A} \mathrm{~m}^{-2} / 25 \mathrm{~m} \mathrm{~s}^{-1}\right.$ in the simulations), the energy barrier is not large enough to compete with the gyrotropic force and the skyrmions are expelled out of the racetrack. ${ }^{65}$ Nevertheless, our simulations show that this threshold can be easily tuned by varying the height of the barrier, for instance by increasing the anisotropy of the pristine film and the irradiation fluence. Indeed, increasing the anisotropy field in the non-irradiated area from $\mu_{0} H_{K}=72 \mathrm{mT}$ to $288 \mathrm{mT}$ increases the maximum velocity from $25 \mathrm{~m} \mathrm{~s}^{-1}$ (black star, Fig. 5(b)) to $65 \mathrm{~m} \mathrm{~s}^{-1}$ (red star), without affecting much the dependence of the velocity on the current density. The maximum velocity for skyrmion motion in the irradiated track increases monotonously with the anisotropy field (Fig. 5(c)). The simulations also show that the magnetic damping has a large impact on the skyrmion dynamics in the irradiated track: decreasing the magnetic damping from 0.3 to 0.01 leads to a large enhancement of the skyrmion velocity (by a factor of about 20, see Fig. 5(b)). However, the maximum velocity to achieve a confined dynamics is little affected by the magnetic damping (around $60 \mathrm{~m} \mathrm{~s}^{-1}$ for $\mu_{0} H_{K}=288 \mathrm{mT}$ in this case). A similar behaviour was reported for the skyrmion dynamics in patterned tracks. ${ }^{33}$

To explain these results, we consider a simple analytical model based on the Thiele equation, ${ }^{66}$ where the skyrmion spin texture is assumed to stay rigid during its motion. Assuming further that the skyrmion radius $(R)$ is large compared to the domain wall width 
$(\Delta)$, the steady-state velocity along the track writes:

$$
v_{\text {track }}=\frac{\pi}{2} \frac{\gamma \Delta}{\alpha} C_{D L} J \cos \chi
$$

where $\chi$ is the angle between the current direction and the track. Thus the skyrmion velocity scales inversely with the damping parameter $\alpha$ in the track. In the absence of track, the velocity reads: 9,17

$$
v_{\text {free }}=\frac{\pi}{4} \frac{\gamma R}{\sqrt{\left(\frac{\alpha R}{2 \Delta}\right)^{2}+1}} C_{D L} J
$$

The gain in velocity in an ion-defined racetrack compared to a confinement-free motion writes $v_{\text {track }} / v_{\text {free }}=\sqrt{1+\tan ^{2} \theta_{S k H}} \cos \chi$, where $\theta_{S k H}$ is the skyrmion Hall angle, i.e the angle between the current and the skyrmion motion direction (without track). For small damping values, $\alpha \ll \Delta / R, v_{\text {track }} / v_{\text {free }} \approx 2 \Delta \cos \chi /(\alpha R)$. For instance, for $R / \Delta \approx 5$ and $\alpha \approx 0.01$, a gain in velocity of around 40 is expected. The velocity in the track can therefore be enhanced by using low-damping materials and by increasing the anisotropy barrier height.

Regarding the influence of the anisotropy barrier, the maximum velocity before the skyrmion leaves the track writes $v_{\max }=F_{\max } / G\left(\frac{\tan \chi}{\tan \theta_{S k H}}-1\right)^{-1}$, where $F_{\max }$ is the maximum lateral force due to the confinement potential and $G$ is the gyrovector. Thus, when the current flows along the track $(\chi=0)$, the maximum velocity depends only on $F_{\text {max }}$, which in our case is set by the difference in anisotropy between the irradiated and non-irradiated regions. It can be enhanced by playing on the angle $\chi$ between the current and the track and as expected it diverges when the track is aligned along the skyrmion Hall angle $\chi=\theta_{S k H}$.

In summary, light-ion-irradiation offers a new method to stabilise skyrmions in tracks and guide their motion with foreseen advantages of lower pinning, both at the edge and within the tracks. In this study, we showed that by locally tailoring the interface PMA and DMI with a focused ion beam, $\mathrm{He}^{+}$-irradiation enables the formation of skyrmions in a film where they would not otherwise be stable. Micromagnetic simulations reproduce well these 
results and confirm that, not only irradiation helps in the stabilisation of skyrmions, but also confines them in designed geometries and channels their motion. The repulsive force from the track edge enhances their longitudinal velocity up to a critical current density above which the skyrmions are expelled from the racetrack. Increasing the depth of the potential well along with using low damping materials would allow to reach even higher skyrmion velocities, free from skyrmion Hall effect. Moreover, $\mathrm{He}^{+}$-irradiation is applicable to virtually

any kind of ultra-thin materials including ferrimagnets, ${ }^{67-69}$ synthetic antiferromagnets ${ }^{70}$ or exchange-biased ferromagnets, ${ }^{71-73}$ for example to channel the skyrmion motion without any external field. This method can also be applied through a mask using a broad ion beam, which makes $\mathrm{He}^{+}$-irradiation compatible with large-scale wafers for applications. In short, this external degree of control offers promising perspectives to further improve control of magnetic skyrmion stability and dynamics in ultra-thin films, giving a significant push towards their technological integration.

\section{Acknowledgement}

We would like to thank Jan Vogel for his help with the focused pMOKE measurements at the Institut Néel in Grenoble, Jordi Prat for his support at the CIRCE beamline, as well as Titiksha Srivastava for fruitful discussions on the formulation of the manuscript. The authors acknowledge the support of the Agence Nationale de la Recherche, project ANR17-CE24-0045 (SKYLOGIC), the French RENATECH network, the support of the DARPA TEE program through grant MIPR\# HR0011831554 from the DOI, and the EU Framework Programme for Research and Innovation HORIZON 2020, project CALIPSOplus under the Grant Agreement 730872.

\section{Supporting Information Available}

The following file is available as supporting information. 
- SI.pdf: This file includes information on the measurement of the Dzyaloshinskii-Moriya interaction by Brillouin light scattering spectroscopy.

\section{References}

(1) Kang, W.; Huang, Y.; Zhang, X.; Zhou, Y.; Zhao, W. Skyrmion-Electronics: An Overview and Outlook. Proc. IEEE 2016, 104, 2040.

(2) Fert, A.; Reyren, N.; Cros, V. Magnetic Skyrmions: Advances in Physics and Potential Applications. Nat. Rev. Mater. 2017, 2, 17031.

(3) Jiang, W.; Upadhyaya, P.; Zhang, W.; Yu, G.; Jungfleisch, M. B.; Fradin, F. Y.; Pearson, J. E.; Tserkovnyak, Y.; Wang, K. L.; Heinonen, O.; te Velthuis, S. G. E.; Hoffmann, A. Blowing Magnetic Skyrmion Bubbles. Science 2015, 349, 283.

(4) Moreau-Luchaire, C. et al. Additive Interfacial Chiral Interaction in Multilayers for Stabilization of Small Individual Skyrmions at Room Temperature. Nat. Nanotech. 2016, $\underline{11}, 444$.

(5) Boulle, O. et al. Room-Temperature Chiral Magnetic Skyrmions in Ultrathin Magnetic Nanostructures. Nat. Nanotech. 2016, 11, 449.

(6) Woo, S. et al. Observation of Room-Temperature Magnetic Skyrmions and Their Current-Driven Dynamics in Ultrathin Metallic Ferromagnets. Nat. Mater. 2016, 15, 501.

(7) Yu, G.; Upadhyaya, P.; Li, X.; Li, W.; Kim, S. K.; Fan, Y.; Wong, K. L.; Tserkovnyak, Y.; Amiri, P. K.; Wang, K. L. Room-Temperature Creation and Spin-Orbit Torque Manipulation of Skyrmions in Thin Films with Engineered Asymmetry. Nano Lett. 2016, 16, 1981. 
(8) Soumyanarayanan, A.; Raju, M.; Oyarce, A. L. G.; Tan, A. K. C.; Im, M.-Y.; Petrović, A. P.; Ho, P.; Khoo, K. H.; Tran, M.; Gan, C. K.; Ernult, F.; Panagopoulos, C. Tunable Room-Temperature Magnetic Skyrmions in Ir/Fe/Co/Pt Multilayers. Nat. Mater. 2017, 16,898 .

(9) Hrabec, A.; Sampaio, J.; Belmeguenai, M.; Gross, I.; Weil, R.; Chérif, S. M.; Stashkevich, A.; Jacques, V.; Thiaville, A.; Rohart, S. Current-Induced Skyrmion Generation and Dynamics in Symmetric Bilayers. Nat. Comm. 2017, 8, 15765.

(10) Legrand, W.; Maccariello, D.; Reyren, N.; Garcia, K.; Moutafis, C.; MoreauLuchaire, C.; Collin, S.; Bouzehouane, K.; Cros, V.; Fert, A. Room-Temperature Current-Induced Generation and Motion of Sub-100 Nm Skyrmions. Nano Lett. 2017, $\underline{17}, 2703$.

(11) Fert, A.; Cros, V.; Sampaio, J. Skyrmions on the Track. Nat. Nanotech. 2013, 8, 152.

(12) Zhang, X.; Ezawa, M.; Zhou, Y. Magnetic Skyrmion Logic Gates: Conversion, Duplication and Merging of Skyrmions. Sci. Rep. 2015, 5, 9400.

(13) Chauwin, M.; Hu, X.; Garcia-Sanchez, F.; Betrabet, N.; Paler, A.; Moutafis, C.; Friedman, J. S. Skyrmion Logic System for Large-Scale Reversible Computation. Phys. Rev. Applied 2019, 12, 064053.

(14) Zhang, X.; Zhou, Y.; Song, K. M.; Park, T.-E.; Xia, J.; Ezawa, M.; Liu, X.; Zhao, W.; Zhao, G.; Woo, S. Skyrmion-Electronics: Writing, Deleting, Reading and Processing Magnetic Skyrmions toward Spintronic Applications. J. Phys.: Condens. Matter 2020, $\underline{32}, 143001$.

(15) Zhang, H.; Zhu, D.; Kang, W.; Zhang, Y.; Zhao, W. Stochastic Computing Implemented by Skyrmionic Logic Devices. Phys. Rev. Applied 2020, 13, 054049. 
(16) Li, S.; Kang, W.; Zhang, X.; Nie, T.; Zhou, Y.; Wang, K. L.; Zhao, W. Magnetic Skyrmions for Unconventional Computing. Mater. Horiz. 2021, Advance Article doi:0.2478/amm-2014-0228.

(17) Juge, R. et al. Current-Driven Skyrmion Dynamics and Drive-Dependent Skyrmion Hall Effect in an Ultrathin Film. Phys. Rev. Appl. 2019, 12, 044007.

(18) Jiang, W.; Zhang, X.; Yu, G.; Zhang, W.; Wang, X.; Benjamin Jungfleisch, M.; Pearson, J. E.; Cheng, X.; Heinonen, O.; Wang, K. L.; Zhou, Y.; Hoffmann, A.; te Velthuis, S. G. E. Direct Observation of the Skyrmion Hall Effect. Nat. Phys. 2017, $\underline{13}, 162$.

(19) Litzius, K. et al. Skyrmion Hall Effect Revealed by Direct Time-Resolved X-Ray Microscopy. Nat. Phys. 2017, 13, 170.

(20) Juge, R. et al. Magnetic Skyrmions in Confined Geometries: Effect of the Magnetic Field and the Disorder. J. Magn. Magn. Mater. 2018, $\underline{455}, 3$.

(21) Büttner, F.; Lemesh, I.; Schneider, M.; Pfau, B.; Günther, C. M.; Hessing, P.; Geilhufe, J.; Caretta, L.; Engel, D.; Krüger, B.; Viefhaus, J.; Eisebitt, S.; Beach, G. S. D. Field-Free Deterministic Ultrafast Creation of Magnetic Skyrmions by Spin-Orbit Torques. Nat. Nanotech. 2017, 12, 1040.

(22) Cayssol, F.; Ravelosona, D.; Chappert, C.; Ferré, J.; Jamet, J. P. Domain Wall Creep in Magnetic Wires. Phys. Rev. Lett. 2004, 92, 107202.

(23) Herrera Diez, L.; Jeudy, V.; Durin, G.; Casiraghi, A.; Liu, Y. T.; Voto, M.; Agnus, G.; Bouville, D.; Vila, L.; Langer, J.; Ocker, B.; Lopez-Diaz, L.; Ravelosona, D. Wire Edge Dependent Magnetic Domain Wall Creep. Phys. Rev. B 2018, 98, 054417.

(24) Jibiki, Y.; Goto, M.; Tamura, E.; Cho, J.; Miki, S.; Ishikawa, R.; Nomura, H.; Srivastava, T.; Lim, W.; Auffret, S.; Baraduc, C.; Bea, H.; Suzuki, Y. Skyrmion Brownian 
Circuit Implemented in Continuous Ferromagnetic Thin Film. Appl. Phys. Lett. 2020, 117, 082402.

(25) Song, M.; Moon, K.-W.; Yang, S.; Hwang, C.; Kim, K.-J. Guiding of Dynamic Skyrmions Using Chiral Magnetic Domain Wall. Appl. Phys. Express 2020, 13, 063002.

(26) Purnama, I.; Gan, W. L.; Wong, D. W.; Lew, W. S. Guided Current-Induced Skyrmion Motion in 1D Potential Well. Sci. Rep. 2015, 5, 10620.

(27) Lai, P.; Zhao, G. P.; Tang, H.; Ran, N.; Wu, S. Q.; Xia, J.; Zhang, X.; Zhou, Y. An Improved Racetrack Structure for Transporting a Skyrmion. Sci. Rep. 2017, 7, 45330.

(28) Fook, H. T.; Gan, W. L.; Purnama, I.; Lew, W. S. Mitigation of Magnus Force in Current-Induced Skyrmion Dynamics. IEEE Transactions on Magnetics 2015, 51, 1-4.

(29) Toscano, D.; Mendonça, J. P. A.; Miranda, A. L. S.; de Araujo, C. I. L.; Sato, F.; Coura, P. Z.; Leonel, S. A. Suppression of the Skyrmion Hall Effect in Planar Nanomagnets by the Magnetic Properties Engineering: Skyrmion Transport on Nanotracks with Magnetic Strips. Journal of Magnetism and Magnetic Materials 2020, 504, 166655.

(30) Bhatti, S.; Piramanayagam, S. N. Effect of Dzyaloshinskii-Moriya Interaction Energy Confinement on Current-Driven Dynamics of Skyrmions. phys. status solidi (rrl) 2019, 13, 1900090.

(31) Loreto, R. P.; Zhang, X.; Zhou, Y.; Ezawa, M.; Liu, X.; de Araujo, C. I. L. Manipulation of Magnetic Skyrmions in a Locally Modified Synthetic Antiferromagnetic Racetrack. Journal of Magnetism and Magnetic Materials 2019, 482, 155-159.

(32) Ang, C. C. I.; Gan, W.; Lew, W. S. Bilayer Skyrmion Dynamics on a Magnetic Anisotropy Gradient. New J. Phys. 2019, 21, 043006.

(33) Iwasaki, J.; Koshibae, W.; Nagaosa, N. Colossal Spin Transfer Torque Effect on Skyrmion along the Edge. Nano Lett. 2014, 14, 4432. 
(34) Lai, P.; Zhao, G. P.; Morvan, F. J.; Wu, S. Q.; Ran, N. Motion of Skyrmions in WellSeparated Two-Lane Racetracks. SPIN 2017, 07, 1740006.

(35) Zhang, X.; Zhao, G. P.; Fangohr, H.; Liu, J. P.; Xia, W. X.; Xia, J.; Morvan, F. J. Skyrmion-Skyrmion and Skyrmion-Edge Repulsions in Skyrmion-Based Racetrack Memory. Sci. Rep. 2015, 5, 7643 .

(36) Yan, Z.; Liu, Y.; Guang, Y.; Feng, J.; Lake, R.; Yu, G.; Han, X. Robust Skyrmion Shift Device Through Engineering the Local Exchange-Bias Field. Phys. Rev. Applied 2020, $\underline{14}, 044008$.

(37) Ang, C. C. I.; Gan, W.; Wong, G. D. H.; Lew, W. S. Electrical Control of Skyrmion Density via Skyrmion-Stripe Transformation. Phys. Rev. Applied 2020, 14, 054048.

(38) Sapozhnikov, M. V.; Vdovichev, S. N.; Ermolaeva, O. L.; Gusev, N. S.; Fraerman, A. A.; Gusev, S. A.; Petrov, Y. V. Artificial Dense Lattice of Magnetic Bubbles. Appl. Phys. Lett. 2016, 109, 042406.

(39) Toscano, D.; Leonel, S. A.; Coura, P. Z.; Sato, F. Building Traps for Skyrmions by the Incorporation of Magnetic Defects into Nanomagnets: Pinning and Scattering Traps by Magnetic Properties Engineering. Journal of Magnetism and Magnetic Materials 2019, 480, $171-185$.

(40) Sapozhnikov, M. V.; Ermolaeva, O. V.; Skorokhodov, E. V.; Gusev, N. S.; Drozdov, M. N. Magnetic Skyrmions in Thickness-Modulated Films. Jetp Lett. 2018, 107, $364-368$.

(41) Chappert, C.; Bernas, H.; Ferré, J.; Kottler, V.; Jamet, J.-P.; Chen, Y.; Cambril, E.; Devolder, T.; Rousseaux, F.; Mathet, V.; Launois, H. Planar Patterned Magnetic Media Obtained by Ion Irradiation. Science 1998, 280, 1919. 
(42) Fassbender, J.; McCord, J. Magnetic Patterning by Means of Ion Irradiation and Implantation. J. Magn. Magn. Mater. 2008, 320, 579.

(43) Fassbender, J.; Ravelosona, D.; Samson, Y. Tailoring Magnetism by Light-Ion Irradiation. J. Phys. D: Appl. Phys. 2004, 37, R179-R196.

(44) Rettner, C. T.; Anders, S.; Baglin, J. E. E.; Thomson, T.; Terris, B. D. Characterization of the Magnetic Modification of $\mathrm{Co} / \mathrm{Pt}$ Multilayer Films by $\mathrm{He}+, \mathrm{Ar}+$, and $\mathrm{Ga}+\mathrm{Ion}$ Irradiation. Appl. Phys. Lett. 2002, 80, 279.

(45) Balk, A. L.; Kim, K.-W.; Pierce, D. T.; Stiles, M. D.; Unguris, J.; Stavis, S. M. Simultaneous Control of the Dzyaloshinskii-Moriya Interaction and Magnetic Anisotropy in Nanomagnetic Trilayers. Phys. Rev. Lett. 2017, 119, 077205.

(46) Diez, L. H.; García-Sánchez, F.; Adam, J.-P.; Devolder, T.; Eimer, S.; Hadri, M. S. E.; Lamperti, A.; Mantovan, R.; Ocker, B.; Ravelosona, D. Controlling Magnetic Domain Wall Motion in the Creep Regime in $\mathrm{He}+-$ Irradiated CoFeB/MgO Films with Perpendicular Anisotropy. Appl. Phys. Lett. 2015, 107, 032401.

(47) Diez, L. H. et al. Enhancement of the Dzyaloshinskii-Moriya Interaction and Domain Wall Velocity through Interface Intermixing in Ta/CoFeB/MgO. Phys. Rev. B 2019, $\underline{99}, 054431$.

(48) Zhao, X. et al. Enhancing Domain Wall Velocity through Interface Intermixing in W-CoFeB-MgO Films with Perpendicular Anisotropy. Appl. Phys. Lett. 2019, 115, 122404 .

(49) Devolder, T.; Barisic, I.; Eimer, S.; Garcia, K.; Adam, J.-P.; Ockert, B.; Ravelosona, D. Irradiation-Induced Tailoring of the Magnetism of CoFeB/MgO Ultrathin Films. J. Appl. Phys. 2013, 113, 203912. 
(50) Nembach, H. T.; Jué, E.; Poetzger, K.; Fassbender, J.; Silva, T. J.; Shaw, J. M. Tuning of the Dzyaloshinskii-Moriya Interaction by $\mathrm{He}+$ Ion Irradiation. 2020, arXiv:2008.06762 [cond-mat.mtrl-sci], https://arxiv.org/abs/2008.06762,

(51) Zhang, S.; Petford-Long, A. K.; Phatak, C. Creation of Artificial Skyrmions and Antiskyrmions by Anisotropy Engineering. Sci. Rep. 2016, $\underline{6}, 31248$.

(52) Fallon, K.; Hughes, S.; Zeissler, K.; Legrand, W.; Ajejas, F.; Maccariello, D.; McFadzean, S.; Smith, W.; McGrouther, D.; Collin, S.; Reyren, N.; Cros, V.; Marrows, C. H.; McVitie, S. Controlled Individual Skyrmion Nucleation at Artificial Defects Formed by Ion Irradiation. Small 2020, 16, 1907450.

(53) Sapozhnikov, M. V.; Petrov, Y. V.; Gusev, N. S.; Temiryazev, A. G.; Ermolaeva, O. L.; Mironov, V. L.; Udalov, O. G. Artificial Dense Lattices of Magnetic Skyrmions. Materials 2020, 13, 99.

(54) Devolder, T.; Ferré, J.; Chappert, C.; Bernas, H.; Jamet, J.-P.; Mathet, V. Magnetic Properties of He+ Irradiated Pt/Co/Pt Ultrathin Films. Phys. Rev. B 2001, $\underline{64}, 064415$.

(55) Cayssol, F.; Menéndez, J. L.; Ravelosona, D.; Chappert, C.; Jamet, J.-P.; Ferré, J.; Bernas, H. Enhancing Domain Wall Motion in Magnetic Wires by Ion Irradiation. Appl. Phys. Lett. 2005, 86, 022503.

(56) Belmeguenai, M.; Adam, J.-P.; Roussigné, Y.; Eimer, S.; Devolder, T.; Kim, J.-V.; Cherif, S. M.; Stashkevich, A.; Thiaville, A. Interfacial Dzyaloshinskii-Moriya Interaction in Perpendicularly Magnetized $\mathrm{Pt} / \mathrm{Co} / \mathrm{AlO}_{\mathrm{x}}$ Ultrathin Films Measured by Brillouin Light Spectroscopy. Phys. Rev. B 2015, 91, 180405.

(57) Devolder, T. Light Ion Irradiation of Co/Pt Systems: Structural Origin of the Decrease in Magnetic Anisotropy. Phys. Rev. B 2000, 62, 5794. 
(58) Casiraghi, A.; Magni, A.; Diez, L. H.; Langer, J.; Ocker, B.; Pasquale, M.; Ravelosona, D.; Durin, G. Bloch-to-Néel Domain Wall Transition Evinced through Morphology of Magnetic Bubble Expansion in Ta/CoFeB/MgO Layers.,2019, arXiv:1907.03708 [cond-mat.mtrl-sci], https://arxiv.org/abs/1907.03708

(59) Zimmermann, B.; Legrand, W.; Maccariello, D.; Reyren, N.; Cros, V.; Blügel, S.; Fert, A. Dzyaloshinskii-Moriya Interaction at Disordered Interfaces from Ab Initio Theory: Robustness against Intermixing and Tunability through Dusting. Appl. Phys. Lett. 2018, 113, 232403.

(60) Debski, A.; Debski, R.; Gasior, W. New Features of Entall Database: Comparison of Experimental and Model Formation Enthalpies. Arch. Metall. Mater. 2014, 59, 1137.

(61) Aballe, L.; Foerster, M.; Pellegrin, E.; Nicolas, J.; Ferrer, S. The ALBA Spectroscopic LEEM-PEEM Experimental Station: Layout and Performance. J. Synchrotron. Rad. 2015, 22,745 .

(62) Foerster, M.; Prat, J.; Massana, V.; Gonzalez, N.; Fontsere, A.; Molas, B.; Matilla, O.; Pellegrin, E.; Aballe, L. Custom Sample Environments at the ALBA XPEEM.

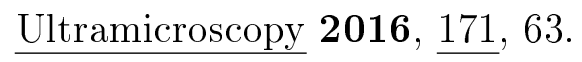

(63) Vansteenkiste, A.; Leliaert, J.; Dvornik, M.; Helsen, M.; Garcia-Sanchez, F.; Waeyenberge, B. V. The Design and Verification of MuMax3. AIP Adv. 2014, 4, 107133.

(64) Sampaio, J.; Cros, V.; Rohart, S.; Thiaville, A.; Fert, A. Nucleation, Stability and Current-Induced Motion of Isolated Magnetic Skyrmions in Nanostructures. Nat. Nanotech. 2013, $\underline{8}, 839$.

(65) Yoo, M.-W.; Cros, V.; Kim, J.-V. Current-Driven Skyrmion Expulsion from Magnetic Nanostrips. Phys. Rev. B 2017, 95, 184423. 
(66) Thiele, A. A. Steady-State Motion of Magnetic Domains. Phys. Rev. Lett. 1973, 30, 230.

(67) Woo, S. et al. Current-Driven Dynamics and Inhibition of the Skyrmion Hall Effect of Ferrimagnetic Skyrmions in GdFeCo Films. Nat. Comm. 2018, 9, 959.

(68) Caretta, L. et al. Fast Current-Driven Domain Walls and Small Skyrmions in a Compensated Ferrimagnet. Nat. Nanotech. 2018, 13, 1154.

(69) Hirata, Y. et al. Vanishing Skyrmion Hall Effect at the Angular Momentum Compensation Temperature of a Ferrimagnet. Nat. Nanotechnol. 2019, 14, 232-236.

(70) Legrand, W.; Maccariello, D.; Ajejas, F.; Collin, S.; Vecchiola, A.; Bouzehouane, K.; Reyren, N.; Cros, V.; Fert, A. Room-Temperature Stabilization of Antiferromagnetic Skyrmions in Synthetic Antiferromagnets. Nat. Mater. 2019, 19, 34.

(71) Yu, G. et al. Room-Temperature Skyrmions in an Antiferromagnet-Based Heterostructure. Nano Lett. 2018, 18, 980.

(72) Rana, K. G.; Finco, A.; Fabre, F.; Chouaieb, S.; Haykal, A.; Buda-Prejbeanu, L. D.; Fruchart, O.; Le Denmat, S.; David, P.; Belmeguenai, M.; Denneulin, T.; DuninBorkowski, R. E.; Gaudin, G.; Jacques, V.; Boulle, O. Room-Temperature Skyrmions at Zero Field in Exchange-Biased Ultrathin Films. Phys. Rev. Appl. 2020, 13, 044079.

(73) Guang, Y. et al. Electron Beam Lithography of Magnetic Skyrmions. Advanced Materials 2020, 32, 2003003. 